\title{
Uncommon Fungi Isolated from Diabetic Patients Toenails With or Without Visible Onychomycoses
}

\author{
Marisela González-Avila • Juan Vicente Gómez-Gómez • \\ Alejandra Paula Espinosa Texis · José Luis Imbert-Palafox • \\ Marco Antonio Becerril-Flores · José Luis Blasco
}

Received: 4 October 2010/ Accepted: 22 March 2011

(C) Springer Science+Business Media B.V. 2011

\begin{abstract}
Kodamaea ohmeri and Prototheca wickerhamii are rare pathogens for humans, and even more rare as cause of onychomycosis. This work reports the second case of onychomycosis by K. ohmeri and the fourth of onycoprotothecosis; it was made in public health institutions in the Hidalgo State, Mexico, studying 261 diabetic patients during 2005 and 2006. Kodamaea ohmeri was isolated from toenails of a 51-year-old female patient, and $P$. wickerhamii from three female patients of 48, 49, and 61 years old, respectively, all of them with type 2 diabetes mellitus (DM 2). Identifications were done by standard
\end{abstract}

M. González-Avila · J. L. Blasco ( $ه)$

Biotechnology Area, Universidad Politécnica de Pachuca, Carretera Pachuca-Ciudad Sahagún Km. 20, Ex Hacienda de Santa Bárbara, Zempoala, Hidalgo 43830, Mexico e-mail: jlblascoc@yahoo.com.mx

J. V. Gómez-Gómez · J. L. Imbert-Palafox ·

M. A. Becerril-Flores

Área Académica de Medicina, Instituto de Ciencias de la Salud, Universidad Autónoma del Estado de Hidalgo,

Carretera Actopan-Tilcuautla, Ex Hacienda de la

Concepción s/n, Hidalgo 42160, Mexico

A. P. Espinosa Texis

Centro de Investigaciones Microbiológicas, Instituto de Ciencias, Benemérita Universidad Autónoma de Puebla, Ciudad Universitaria, Puebla 72570, Mexico

M. González-Avila

Centro de Investigación y Asistencia en Tecnología y Diseño de Jalisco A. C., Av. Normalistas $N^{\circ}$. 800, Colinas de la Normal, Guadalajara, Jalisco 44270, Mexico microbiological methods and a commercial system. Only one patient infected with $P$. wickerhamii showed mixed infection with dermatophytes. Out of the total studied DM 2 patients, $1.15 \%$ presented onycoprotothecosis and $0.38 \%$ onychomycosis by K. ohmeri, high percentages if it is considered that few cases have been reported of $K$. ohmeri and $P$. wickerhamii as onychomycosis causal agents.

Keywords Prototheca wickerhamii . Kodamaea ohmeri · Onychomycoses . Diabetic patient . Yeast-like

\section{Introduction}

Diabetic patients may present with complications involving all systems of the body [1]. Diabetic foot represents a major risk for foot amputation [2]; fungal nail infection can contribute to the severity of diabetic foot [3].

Fungal infection in toenails may be a major cause of morbility in feet of diabetic patients, and the presence of mycotic nail may result in infection to the adjacent nail or skin injury and provide a reservoir of pathogen fungi [4]. Causal agents responsible for onychomycoses can be dermatophytes, yeasts, and molds; the agents most frequently isolated from toenails are dermatophytes in both diabetic and non-diabetic patients [1]. In contrast, Dogra et al. [5] reported yeasts were 
most common causal agents for toenail onychomycoses in diabetic patients in India. Although there are uncommon causal agents of onychomycoses, among them yeasts as Kodamaea ohmeri (Pichia ohmeri) and yeast-like organisms such as Prototheca wickerhamii are extremely rare [6].

Kodamaea ohmeri is an ascomycetous yeast formerly named Pichia ohmeri according to Yamada et al. [7]. It has been reported as a causal agent of fungemia mainly in pediatric patients [8,9], associated with surgical processes which needed use of catheters $[10$, 11] and in case of urinary tract infection [12]. There is only one case reported for onychomycosis caused by $K$. ohmeri; it was isolated from a patient with type 2 diabetes mellitus (DM 2) in Mexico City [13].

On the other side, reported cases of onycoprotothecosis are rare. Genus Prototheca are achlorophyllic algae, and their natural habitats are plants [14]. Moreover, these organisms have been more commonly isolated in catheters, dogs and cats guts, and as mastitis causal agent [15-18]. Protothecoses appear habitually by traumatic inoculation and usually in immunocompromised patients [19]. Overall, pathogenicity and virulence are moderate, and Prototheca species are considered rare opportunistic pathogens [20]. There are two reported cases of onychoprotothecoses caused by $P$. zopfii $[21,22]$ and three by $P$. wickerhamii [23-25]. Present work describes rare causal agents found in toenails from diabetic patients.

\section{Materials and Methods}

\section{Materials}

$\mathrm{KOH}, \mathrm{NaOH}$, glucose, and peptone were purchased from JT Baker Mexico (Mexico DF, Mexico); Sabouraud-chloramphenicol media BBL ${ }^{\mathrm{TM}}$, Mycosel agar (cycloheximide and chloramphenicol) $\mathrm{BBL}^{\mathrm{TM}}$, and Corn Meal Agar with Polysorbate $80 \mathrm{BBL}^{\mathrm{TM}}$ were purchased from Beckton and Dickinson Mexico SA (Mexico DF, Mexico), and Candida ID2 chromogenic medium and System API 20C AUX from Biomerieux Mexico SA (Mexico DF, Mexico).

\section{Patients and Sample Taking}

A study was done to determine onychomycosis incidence in diabetic patients. A questionnaire, specifically designed for this work, was applied to 261 patients of both sexes attended in public health institutions of the center-south area in the Hidalgo State, Mexico, in 2005 and 2006. The independent variables that were measured through the transversal, descriptive and observational study design, were: age, sex, body mass index, rural or urban area, education, occupation, smoking, alcoholism, coexistence with animals, shoe type, electricity, water, drainage and type of floor at their houses, baths per week, immunosuppressors and antimycotics intake, cancer, HIV, systemic arterial hypertension, DM type, peripheric neuropathy, and diabetic foot.

Medical exploration was also done in order to detect tinea pedis and/or onychomycosis. To take the nail specimen, both feet were revised and the sample was taken with a decontaminated nail clipper, after asepsis of the nail with more ungueal abnormality. This sample was placed in duplicate in sterile Eppendorf tubes for microbiological diagnosis.

\section{Cultures and Identification of Microorganisms}

Direct examination was done clarifying the sample with $30-40 \% \mathrm{KOH}$ or $10-20 \% \mathrm{NaOH}$; a drop of the clarifying mixture was added to the sample and, it was let stand for $30 \mathrm{~min}$ and then observed with $40 \times$ magnification. Samples that showed yeasts or yeastlike organisms were placed in solid media: $2 \%$ glucose, $1 \%$ peptone, or Sabouraud media with $0.05 \%$ chloramphenicol, and incubated at $30^{\circ} \mathrm{C}$ from 14 to 28 days. To identify yeasts, isolates from Sabouraud media were cultured in ChromAgar ${ }^{\mathrm{TM}}$ media and were done with commercial System API 20C AUX (Biomerieux) [26]. The system consists of a disposable plastic strip with 20 cupules containing dehydrated reagents sufficient for 18 biochemical tests, a negative control and a cupule containing glucose that serves as a positive control. A profile number based upon the reactions observed was generated for each isolate. Identifications were made by reference to the API Analytical Profile Index.

\section{Statistical Analyses}

The statistical analyses were performed with the software "Statistical Package for Social Studies" (SPSS) 12.0 version, using odds ratio, and Chi-square and Mann-Whitney $U$ proofs. 


\section{Results}

Kodamaea ohmeri grew only in Sabouraud media, it did produce germinal tubule and chlamydioconidia, and the color in ChromAgar was yellow-violet. Prototheca wickerhamii grew in Sabouraud agar, but not in mycobiotic agar, and it did not induce germinal tube nor clamidoconidia in maize flour; the cream color developed in ChromAgar has already been reported (Table 1). The profile number obtained with the API 20C AUX for P. wickerhamii was 2040040 and 6146175 for $K$. ohmeri. The results obtained in the antimycotic test performed for these microorganisms are shown in Table 1.

Kodamaea ohmeri was isolated from a 51-year-old female patient that clinically shows total dystrophic onychomycosis not related with dermatophytosis, without treatment (Fig. 1). Clinical history shows DM 2 treated with oral hypoglucemiants. Other clinical features of the patient are shown in Table 2.

Prototheca wickerhamii was isolated from three female patients designated as 1, 2, and 3. All of them were asymptomatic for typical onychomycosis and have in common the age rank, more than 45 (48, 49, and 61 years old, respectively). The three of them showed chronical immunologic compromise by DM 2 , but they have treatment with oral hypoglucemiants and none of them with immunosuppressors. It is relevant to mention that they are from different ecological environments, altitudes, climates, and they have different habits and economical positions (Table 2). Nevertheless, patients 2 and 3 were unrelated with dermatophytic infection (Fig. 2), while patient 1 was positive for mixed infection. The pathological records of patients 2 and 3 showed systemic arterial hypertension, and only patient 2 shows neuropathic damage. All patients cohabit with dogs and patients 1 and 3 with cats additionally (Table 2).

With regard to the entire study of the 261 nail samples of diabetic patients, onychomycosis was caused by Candida guilliermondii (4.6\%), C. parapsilosis (3.4\%), C. glabrata (0.8\%), C. krusei (0.4\%), Candida spp. (1.5\%), K. ohmeri (0.38\%), $P$. wickerhamii $(1.15 \%)$, and a non-identified yeast $(2.7 \%)$.

The statistical analyses of the complete study using odds ratio with $95 \%$ confidence interval (CI) showed, in concordance with Chi-square and Mann-

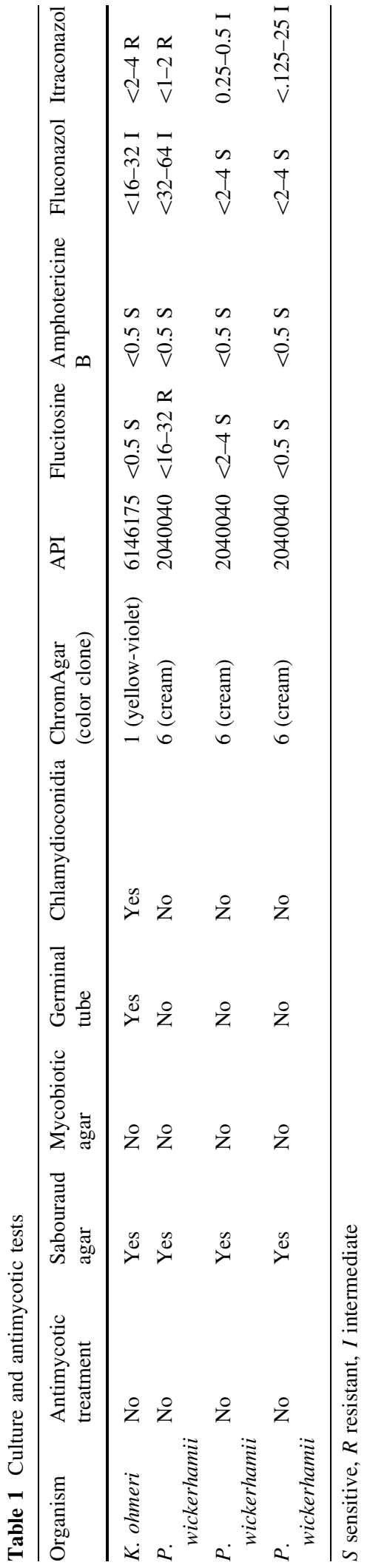




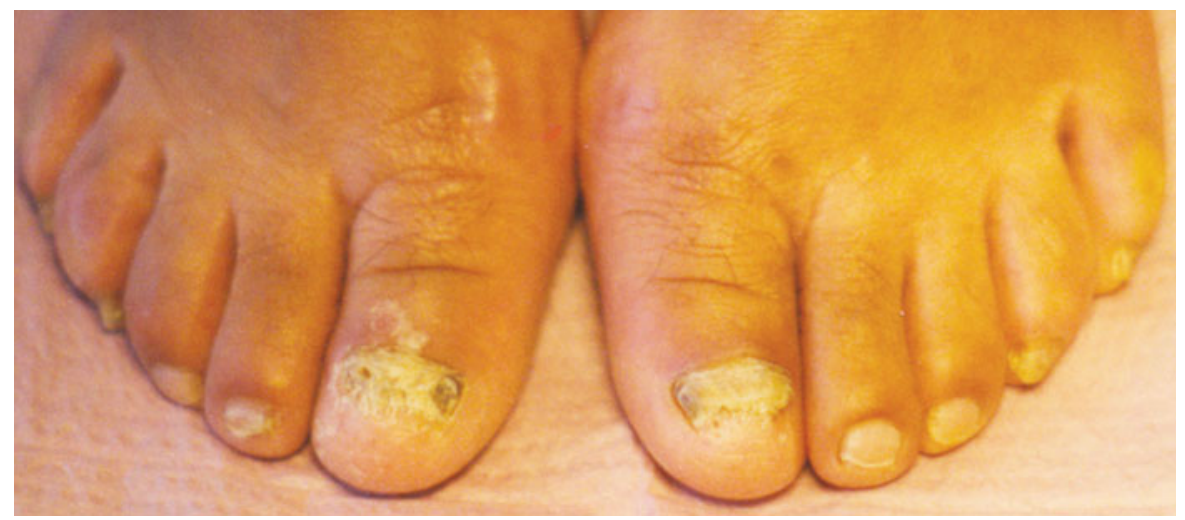

Fig. 1 Complete onycolysis of the first knuckles in both feet in a DM2 female patient caused by K. ohmeri. Ungual destruction is from distal to proximal part but painless; whitish thickening and desquamation are also observed

Whitney $U$ tests, that persons that live in homes with more than one inhabitant per room have 2.43 (CI $95 \%$ 1.21-4.89) more probability of being diagnosed with general onychomycosis than inhabitants in homes without overcrowding. Also, those who are technicians and professionals have 9.42 (CI 95\% 1.52-58.44) times more probabilities of being diagnosed with yeast onychomycosis than those who are farmers, workers, retired, or work at home or in commerce. On the other hand, regarding with yeast onychomycosis, it was found that persons that use open shoe or don't use shoes have 2.59 (CI 95\% 1.14-5.88) times more probabilities of being diagnosed with yeast onychomycosis than those who use close shoe, and persons who are technicians and professionals have 10.49 (1.59-69.11) times more probabilities of having yeast onychomycosis than the ones that have the other occupations. As well, persons with alcohol intake have 3.72 (1.11-12.41) times more probabilities of suffering from yeast onychomycosis than who don't do it. It was observed too that the confidence interval is significative, but very wide, in the occupation variable. The logistic regression made with other methods, always fitted in with these results (data not shown).

\section{Discussion}

In this work, we report one case of onychomycosis by $K$. ohmeri, and three cases of onycoprotothecoses, all in women with DM 2, ranging from 48 to 61 years old. The majority of patients that show fungemia by $K$. ohmeri are pediatric ones [9], and since the first case of a $K$. ohmeri-related infection described in 1998, only 20 cases so far have been reported [13, and reviewed in 9], then this case represents number 21. Only three cases of fungemia caused by $K$. ohmeri appeared in diabetic patients, one was the first $K$. ohmeri-related infection described, in a 48-year-old female with kidney transplant, in USA [27], the second one was associated with chronic renal failure in an 82-year-old female in Spain [11], and the third one is the unique reported case of onychomycosis by $K$. ohmeri, specifically in Mexico [13], being this case the only one in the literature clinically related with the discussed case. The reported patient showed distal-lateral subungual onychomycosis, and mixed infection with Trichophyton rubrum was observed [13]. Therefore, the present case represents the second one of onychomycosis by $K$. ohmeri in Mexico, and in the whole world.

Regarding with onycoprotothecosis, three cases from 261 patients in this study represent $1.15 \%$; this percentage is high if it is considered that few cases have been reported, when $P$. wickerhamii is the causal agent. Human protothecoses are rare, but human onycoprotothecoses by $P$. wickerhamii are extremely rare; only three cases have been reported [23-25]. The natural reservoir of Prototheca are plants, soil, sewage, and fresh water environs [20], and it is interesting to find these organisms infecting toenails in humans, particularly in women in climacteric or postmenopausic stages. Economic activity or habits are not directly related with infection, only patient 2 is in risk because she is farmer, and protothecoses have been reported in fishermans, workers in rice paddies, aquarium staff, and farmers 


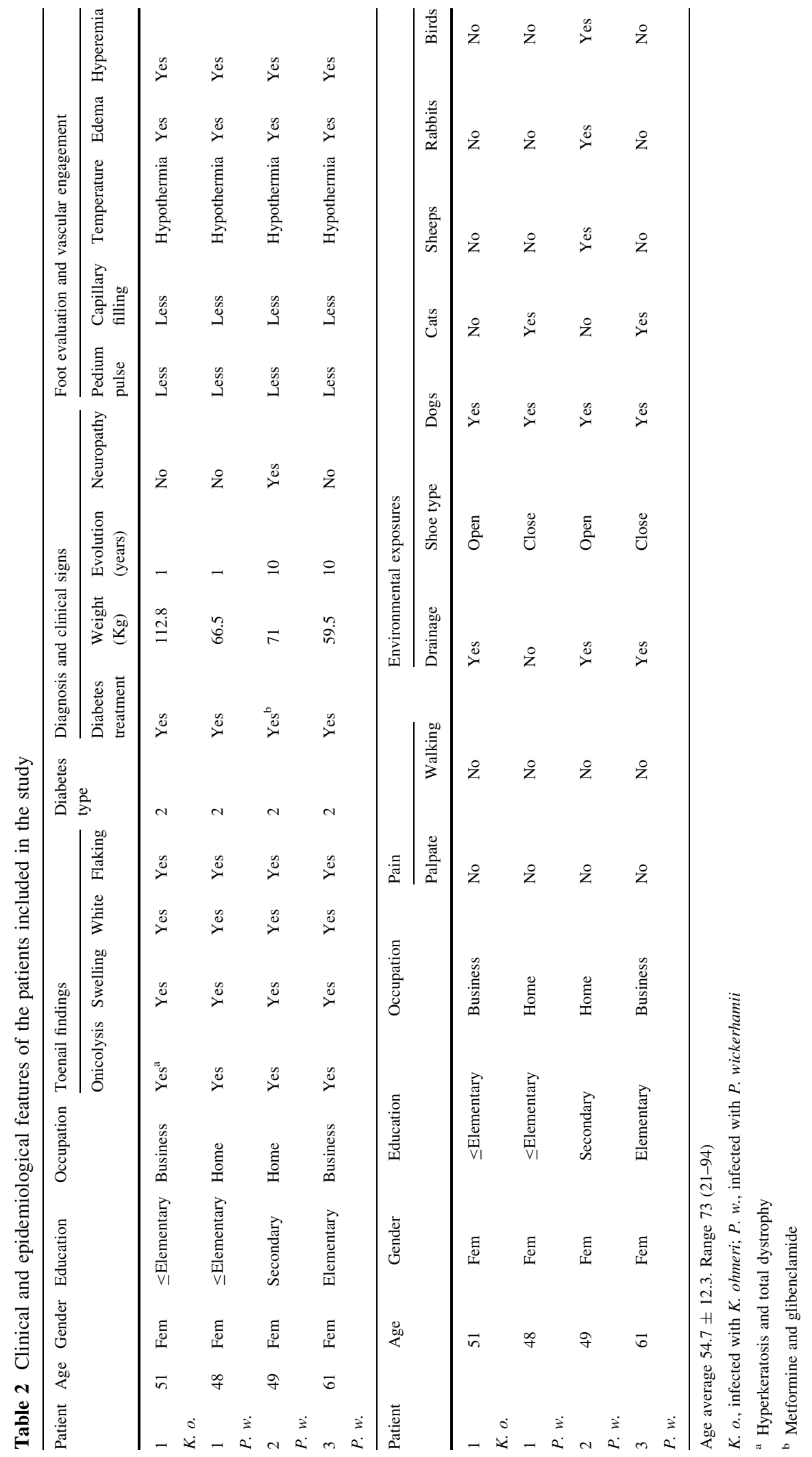




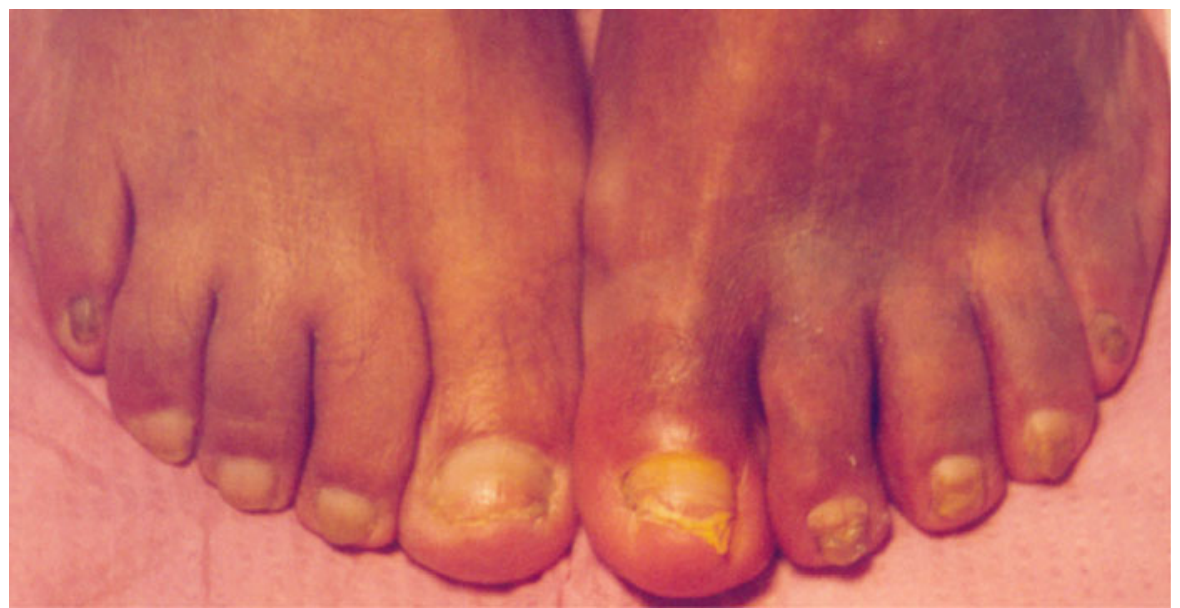

Fig. 2 Partial onycolysis of the first knuckles in feet in a DM2 female patient caused by $P$. wickerhamii. Destruction in distal part of both nails is observed. No pain to palpation was noticed, but it was detected typical symptomatology of vascular complication, mainly in the left foot, observing slight edema, hyperemia and delay in capillary filling, as well as decrease of feet pulses
[14]. The three infected patients referred, cohabit with dogs, and there are reports of systemic protothecosis in dogs, in which organisms of the genus Prototheca have been identified, in guts and feces, where it is relatively frequent compared with human feces [18].

Normally, the described cases of human protothecoses occur in immunocompromised patients [19]. Moreover, onycomycoses are habitually caused by dermatophytes [1] and oddly, only one patient in this study showed mixed infection; this indicates that $P$. wickerhamii does not grow necessarily associated with dermatophytes, according to Galán et al. [23] and Zaitz et al. [25]; they reported cases of onycoprotothecoses in which only $P$. wickerhamii was isolated. It is relevant to mention that at first, skin was the tissue more frequently reported as involved in Prototheca infections [14], and now it is been described associated with surgeries, being the first case reported in 1997 [23], protothecoses are most frequently reported and onychoprototecoses in immunocompromised patients too. This situation requires attention because maybe the ubiquity of these organisms is the result of adaptation processes derived from survival needs.

Acknowledgments This work was supported by a Grant (SEP-PROMEP UAEH CA-25) from the Secretaría de Educación Pública, Mexico.

\section{References}

1. Mayser P, Freund V, Budihardja D. Toenail onychomycosis in diabetic patients. Am J Clin Dermatol. 2009; 10: 211-20.

2. Gupta AK, Humke S. The prevalence and management of onychomycosis in diabetic patients. Eur J Dermatol. 2000; 10:379-84.

3. Rich P, Hare A. Onychomycosis in a special patient population: focus on the diabetic. Int J Dermatol. 1999;38: 17-9.

4. Piérard GE, Piérard-Franchimont $\mathrm{C}$. The nail under fungal siege in patients with type II diabetes mellitus. Mycoses. 2005;48:339-42.

5. Dogra S, Kumar B, Bhansali A, Chakrabarty A. Epidemiology of onychomycosis in patients with diabetes mellitus in India. Int J Dermatol. 2002;41:647-51.

6. Hazen KC. New and emerging yeasts pathogens. Clin Microbiol Rev. 1995;8:462-78.

7. Yamada Y, Suzuki T, Matzuda M, Mikata K. The phylogeny of Yamadazyma ohmeri (Etchells et Bell) BillonGrand based on the partial sequences of $18 \mathrm{~S}$ and $26 \mathrm{~S}$ ribosomal RNAs: the proposal of Kodamaea gen. nov. (Saccharomycetaceae). Biosci Biotechnol Biochem. 1995; 59:1172-4.

8. Otag F, Kuyucu N, Erturan Z, et al. An outbreak of Pichia ohmeri infection in the paediatric intensive care unit: case reports and review of the literature. Mycoses. 2005;48: 265-9.

9. De Barros JD, Do Nascimento SM, De Araújo FJ. Kodamaea (Pichia) ohmeri fungemia in a pediatric patient admitted in a public hospital. Med Mycol. 2009;47:775-9.

10. Han XY, Tarrand JJ, Escudero E. Infections by the yeast Kodamaea (Pichia) ohmeri: two cases and literature review. Eur J Clin Microbiol Infect Dis. 2004;23:127-30. 
11. García-Tapia A, García-Agudo R, Marín P, Conejo JL, García-Martos P. Kodamaea ohmeri fungemia associated with surgery. Rev Iberoam Micol. 2007;24:155-6.

12. Puerto JL, García-Martos P, Saldarreaga A, et al. First report of urinary tract infection due to Pichia ohmeri. Eur J Clin Microbiol Infect Dis. 2002;21:630-1.

13. Manzano-Gayosso P, Hernández-Hernández F, MéndezTovar LJ, et al. Onychomycosis incidence in type 2 diabetes mellitus patients. Mycopathologia. 2008;166:41-5.

14. Lass-Flörl C, Mayr A. Human protothecosis. Clin Microbiol Rev. 2007;20:230-42.

15. Boyd A, Langley M, King L. Cutaneous manifestations of Prototheca infections. J Am Acad Dermatol. 1995;32: 758-64.

16. Marques S, Silva E, Carvalheira J, Thompson G. In vitro antimicrobial susceptibility of Prototheca wickerhamii and Prototheca zopfii isolated from bovine mastitis. J Dairy Sci. 2006;89:4202-4.

17. Pérez-Melón C, Camba M, Tinajas A, et al. Prototheca wickerhamii peritonitis in patients on peritoneal dialysis. Nefrología. 2007;27:81-2.

18. Stenner VJ, Mackay B, King T, et al. Protothecosis in 17 Australian dogs and a review of the canine literature. Med Mycol. 2007;45:249-66.

19. Leimann B, Monteiro P, Lazera M, Candanoza E, Wanke B. Protothecosis. Med Mycol. 2004;42:95-106.
20. Huerre M, Ravisse P, Solomon H, et al. Human protothecosis and environment. Bull Soc Pathol Exot. 1993;86: 484-8.

21. Magerman K, Gordts B, Hindryckx P, Van Landuyt HW. Isolation of Prototheca zopfii from a finger. Case report and review of the literature. Acta Clin Belg. 1991;46: 233-6.

22. Marcano C, Feo M. Prototheca zopfii colonizing the nail. Mycopathologia. 1981;75:89-92.

23. Galán F, García-Martos P, Palomo M, et al. Onychoprotothecosis due to Prototheca wickerhamii. Mycopathologia. 1997;137:75-7.

24. Kam KM, Au WF, Wong PY, Cheung MM. Onychomycosis in Hong Kong. Int J Dermatol. 1997;36:757-61.

25. Zaitz C, Godoy AM, de Sousa VM, et al. Onycoprotothecosis: report of the first case in Brazil. Int $\mathrm{J}$ Dermatol. 2006;45:1071-3.

26. Quindós G, Vargas RA, Helou S, et al. Evaluación micológica de un nuevo medio de cultivo cromógeno (Candida ID $^{\circledR}$ ) para el aislamiento e identificación presuntiva de Candida albicans. Rev Iberoam Micol. 2001;18:23-8.

27. Bergman MM, Gagnon D, Doern GV. Pichia ohmeri fungemia. Diagn Microbiol Infect Dis. 1998;30:229-31. 